\title{
Small fiber neuropathy as a part of fibromyalgia or a separate diagnosis?
}

Fibromyalgia (FM) is a chronic disease characterized mainly by widespread pain, fatigue and cognitive disorders. Small Fiber Neuropathy (SFN) is a generalized sensory nerve disorder with structural and functional nerve abnormalities manifesting themselves as sensory disorders such as allodynia, burning, disturbances in thermal sensation, numbness, paresthesia and hyperesthesia. Small fiber neuropathy may also manifest itself with the restless leg syndrome, dry eyes and mouth, gastroenteric symptoms, problems with bladder control, palpitations or syncope. Clinical features of small fiber neuropathy are also presented by some of fibromyalgia patients. The SFN diagnostics is essentially simple and based on symptom analysis and skin punch biopsy assessment. The confirmation of SFN in fibromyalgia patients may influence their treatment. In this article we present a problem of diagnosis and treatment of small fiber neuropathy; we also discuss a link between fibromyalgia and this type of neuropathy.

Keywords: fibromyalgia $\cdot$ small fiber neuropathy $•$ punch biopsy

\section{Introduction}

Fibromyalgia (FM) is a chronic medical condition characterized by multiple symptoms such as: widespread pain, fatigue, waking unrefreshed and cognitive disorders. Although fibromyalgia occurs in all populations worldwide and symptom prevalence ranges between $2 \%$ and $4 \%$ in the general population [1], the definition, pathogenesis and treatment of the disease are still controversial and its socio-economical aspect is often underestimated. The fibromyalgia ACR 2010 criteria employ a self-report questionnaire (Fibromyalgia Survey Questionnaire FSQ) to assess patient symptoms and to make a diagnosis. As the FM's criteria remain very subjective, during the last few years serious investigators of fibromyalgia realize the profound implications of finding features of Small Fiber Neuropathy (SFN) in this disorder [2].

The Small Fiber Neuropathy (SFN), together with the large fiber neuropathy, belongs to a group of diseases known as peripheral neuropathies. The polyneuropathy is a term used in a case of only large fiber or both large and small fiber involvement, whereas the term small fiber neuropathy covers isolated small fiber involvement [3]. The characteristics of all sensory nerve fibers are presented in Table 1.

SFN is a generalized sensory nerve disorder with structural and functional nerve abnormalities [3]. SFN is histopathologically characterized by the degeneration of small nerve fiber endings. Although the clinical diagnostic criteria for SFN exist, there is no common standard for them.
The small fiber neuropathy is the effect of peripheral nerves damage, including that of small myelinated fibers $A \delta$ and unmyelinated C fibers $[4,5]$. Small nerve fibers are responsible for somatic and autonomic functions. Normally they control pain, heat and cold perception, as well as enteric and autonomic functions. Patients with SFN can present numerous symptoms that include allodynia, burning, lower thermal sensation, numbness, paresthesia, hyperesthesia, abnormal sweating, restless leg syndrome, dry eyes and mouth, gastroenteric symptoms, problems with bladder control and cardiac manifestations like palpitations or syncope [4]. Many symptoms worsen at night and during periods of rest [6].

\section{Ethiopathogenesis and epidemiology of SFN}

The small fiber neuropathy may be present in a variety of metabolic, infectious, inflammatory, toxic and genetic disorders. Some cases seem to be idiopathic [5]. The main metabolic cause of SFN is diabetes mellitus. It is estimated that $16 \%-20 \%$ of diabetic patients have SFN [7]. Glucose intolerance and metabolic syndrome are also associated with SFN. Other metabolic causes include vitamin B12 deficiency, hypervitaminosis B6, chronic kidney disease and hypothyroidism [1,7]. There are a few cases described of patients with progressive burning pain, numbness, tingling, and weakness in a stocking-glove distribution, who were found to have severe pyridoxine toxicity [8]. Among infectious causes of SFN Epstein-Barr virus infection, Lyme disease, hepatitis C, HIV infection and leprosy should be mentioned.

\author{
Marta Swiecka, Maria \\ Maslinska* \& Brygida \\ Kwiatkowska \\ National Institute of Geriatric, \\ Rheumatology and Rehabilitation, Warsaw, \\ Poland \\ ${ }^{*}$ Author for correspondence: \\ maslinskam@gmail.com \\ ORCID ID: https://orcid.org/0000-0002 \\ 2211-0302
}




\begin{tabular}{|c|c|c|c|c|}
\hline $\begin{array}{l}\text { Type of } \\
\text { sensory fiber }\end{array}$ & $\begin{array}{l}\text { Presence of } \\
\text { myelinated axons }\end{array}$ & $\begin{array}{l}\text { Diameter } \\
(\mu \mathrm{m})\end{array}$ & Sensory information conveyed & $\begin{array}{l}\text { Usefulness of } \\
\text { electroneuromyography }\end{array}$ \\
\hline $\mathrm{Aa}$ & Yes & $13-20$ & Proprioception & Yes (H reflex) \\
\hline$A \beta$ & Yes & $6-12$ & $\begin{array}{l}\text { Discriminative sensitivity to mechanical } \\
\text { stimuli (touch, vibration) }\end{array}$ & $\begin{array}{l}\text { Yes (sensory nerve } \\
\text { conduction) }\end{array}$ \\
\hline$A \delta$ & Yes & $1-5$ & $\begin{array}{l}\text { Sensitivity to cold and pain ("rapid" } \\
\text { pain, pinprick) }\end{array}$ & No \\
\hline C & No & $0.3-1.5$ & $\begin{array}{l}\text { Sensitivity to heat and pain ("slow" } \\
\text { pain, burning sensations) }\end{array}$ & No \\
\hline
\end{tabular}

Taking into consideration the chronic inflammatory conditions and connective tissue diseases, the most common causes of SFN are: Primary Sjögren's syndrome, sarcoidosis and systemic lupus erythematous [1]. The main drugs and toxic agent responsible for SFN includes alcohol (although disease progresses to involve the large nerve fibers as well), anticancer agents, such as platinum and bortezomib, isoniazid, metronidazole, antiretroviral drugs [5]. Among genetic causes Fabry's disease and familial amyloid neuropathy due to a transthyretin (TTR) gene mutation should be considered. In Table 2 are presented the most common causes of SFN.

\section{Diagnosis of SFN}

Unmyelinated $\mathrm{C}$ and thinly myelinated $\mathrm{A} \delta$ nerve fibers represent the majority of peripheral sensory nerves in mammals and it is not obvious, how to diagnose their damage. Characteristic for patients with pure small-fiber neuropathy are normal results of neurological and physical examination. In the reflex, motor and coordination examinations no abnormalities are found. Additionally, in some cases, proprioception, light touch are vibratory sensation are also normal. The hallmarks of SFN are decreased pinprick and thermal sensation or hyperalgesia in the affected region and slightly decreased vibratory sensation in some patients [6].

Examinations used for SFN diagnosis are: Quantative Sensory Testing (QTS), Quantitative Sudomotor Axon Reflex Testing (QSART), Skin Biopsy and Electromyography and Nerveconduction Studies [6].

Quantative Sensory Testing (QTS) is an extension of the physical examination [6,9]. It is a valuable method for diagnosing peripheral nervous system disorders. QST essentially determines the sensation and pain thresholds for cold and warm temperatures, as well as for the vibration sensation, by stimulating the skin and comparing the results to normative values. It is a non-invasive and pain-free technique. There are some well-recognized limitations to QST; abnormalities in either the central or peripheral nervous system can result in the same deficit. What is more, QST requires conscious reaction from the patient and in conditions of cognitive disfunctions (e.g. in older patients) the reliability of the test results may be questionable.

The Quantitative Sudomotor Axon Reflex Test (QSART) is one of autonomic function tests. It evaluates the peripheral sympathetic cholinergic nervous system [10]. The test measures the response of autonomic nerves that control sweating. During the test, acetylcholine is introduced by iontophoresis into the skin, directly stimulating sweat glands, with the volume of produced sweat measured. Some patients with the small fiber neuropathy have increased sweat production.

Electromyography and nerve-conduction studies are well-established neurophysiologic techniques, that often produce normal results in pure smallfiber neuropathies, because they allow to assess the damage of large myelinated axons (i.e. reduced sensory action potential amplitude) and myelin sheath (i.e. reduced conduction velocity) only [5].

All the methods mentioned above have their limitations and making the diagnosis of SFN is challenging in clinical practice. That is why during the last 15 years a punch skin biopsy is more often used as a "gold standard" to diagnose SFN and it seems to be the best tool to confirm SFN diagnosis [11]. The punch biopsy allows evaluating a morphometric and qualitative evaluation of somatic and autonomic small nerve fibers.

This method doesn't have the limitations, that neurophysiologic test have and can objectively detect the damage of small fibers. It will be detailly described and discussed later. 


\begin{tabular}{|c|c|}
\hline \multirow{7}{*}{ Metabolic disorders } & Diabetes mellitus \\
\hline & Glucose intolerance \\
\hline & Vitamin $\mathrm{B} 12$ deficiency \\
\hline & Hypothyroidism \\
\hline & Hiperlipidemia \\
\hline & Hipervitaminosis B6 \\
\hline & Chronic kidney disease \\
\hline \multirow{4}{*}{ Dysimmunity/inflammatory diseases } & Sjögren's syndrome \\
\hline & Sarcoidosis \\
\hline & Systemic lupus erythematosus \\
\hline & Celiac disease \\
\hline \multirow{5}{*}{ Infectious } & HIV \\
\hline & Hepatitis C \\
\hline & Ebstein-Barr virus \\
\hline & Lyme disease \\
\hline & Leprosy \\
\hline \multirow{4}{*}{ Toxic agents and medications } & Alcohol \\
\hline & $\begin{array}{l}\text { Antibiotics (metronidazole, nitrofurantoin, linezolid, } \\
\text { isonizid) }\end{array}$ \\
\hline & Anticancer agents (bortezomib, platin) \\
\hline & Antiretroviral drugs \\
\hline \multirow{2}{*}{ Genetic diseases } & Fabry's disease \\
\hline & Familial amyloid polyneuropathy (transthyretin) \\
\hline
\end{tabular}

\section{Current classification criteria for FM}

Current criteria for FM were established in 2010 by American College of Rheumatology (ACR). Since 2010 diagnosis is based only on a self-report questionnaire (Fibromyalgia Survey Questionnaire FSQ) and there is no need for the tender point examination, as it was previously specified in the ACR 1990 classification criteria $[12,13]$. To diagnose FM three conditions must be satisfied:

- Widespread Pain Index (WPI) $\geq 7 / 19$ and Symptom Severity Scale (SSS) Score $\geq 5 / 12$ or WPI between 3-6/19 and SSS $\geq 9 / 12$

- Symptoms being present at a similar level for at least 3 months

- The patient does not have another disorder that would otherwise sufficiently explain the pain. The conditions 1 and 2 are assessed by the Fibromyalgia Survey Questionnaire.

- The link between small fiber neuropathy and widespread pain syndromes

Oaklander et al. tested the hypothesis, whether the acquired small-fiber polyneuropathy (SFPN) contributes to the widespread pain syndromes in pediatrics [14]. They evaluated forty-one patients with unexplained widespread pain that started before the age of 21, with the objective diagnostic tests for SFPN (including neurodiagnostic skin and nerve biopsy, autonomic function testing). The results gathered on poly-ethnic group proved definite SFPN in 59\% of the children, probable small fiber polyneuropathy in $17 \%$, and possible diagnosis of SFPN in $22 \%$.

This result encouraged researchers to perform further studies. Also in 2013 journal "Pain" published the article of Oaklander et al., which showed the outcomes of studies conducted on fibromyalgia patients [15]. They hypothesized, that patients suffering from fibromyalgia have SFPN, which is causing their illness symptoms. In this work, 27 fibromyalgia patients and 30 matched normal controls were examined. All fibromyalgia subjects had to satisfy ACR criteria plus present evidence of a physician's diagnosis. As much as $41 \%$ of distal-leg neurodiagnostic skin biopsies from fibromyalgia group vs. $3 \%$ of biopsies from control subjects were diagnostic for SFPN. Additionally, study instruments also included Michigan Neuropathy Screening Instrument (MNSI), the Utah Early Neuropathy Scale (UENS) and Autonomic-Function Testing (AFT). The results of patients' questionnaires and physical assessments were higher for FM group and there were equally prevalent abnormalities in AFT in both groups.

After 2013 publications about the possible pathogenesis of fibromyalgia became numerous. Several controlled studies describing a decreased 
amount of small nerve fiber density in fibromyalgia patients reinforced the assumption, that fibromyalgia may be a neuropathic pain syndrome. Caro et al. published the results of the studies on forty-one patients with fibromyalgia and forty-seven control subjects that underwent 3-mm skin biopsy at the proximal thigh and distal part of the leg, near the ankle and the epidermal nerve fiber density was analyzed. The researchers excluded all the patients with clinical evidence of small fiber neuropathy [16]. In the results, the epidermal nerve fiber density of FM patients was lower, than that of control patients, at both the thigh and the calf. Also, there was a negative correlation between ENFD at the calf and the age in FM group, but not in control subjects. This result can suggest the accelerated nerve loss in patients with FM. Giannoccaro et al. [17] studied 20 subjects with fibromyalgia. Patients underwent neurological examination, nerve conduction studies, and skin biopsies from distal part of the leg and thigh. In all patients electrodiagnostic studies produced normal results. The epidermal nerve fiber density was reduced in 6 patients and the authors concluded that there is a subset of FM patients that has small fiber neuropathy and that is why skin biopsy should be considered as a diagnostic tool.

Another study conducted by Kosmidis et al. [18] compared Intra Epidermal Nerve Fiber Density (IENFD) in forty-six patients, with the diagnosis of FM according to the ACR 2010 criteria, and thirty-four healthy controls. All the patients underwent $5 \mathrm{~mm}$ punch biopsies. In these results 15 of $46(32.6 \%)$ FM patients had reduced IENFD compared to controls.

\section{Small fiber neuropathy in FM patients}

The research papers mentioned above are just examples of studies confirming small fiber neuropathy in FM patients. In multiple investigative centers peripheral tissue lesion has been reported in those patients. It is assumed, that $50 \%$ of FM patients have SFN [19]. In most of the studies the reduced epidermal fiber density is considered as a sine qua non condition of small fiber neuropathy. Reduced ENFD is often defined as $\leq 5$ th percentile of ENFD values measured in a healthy group [2]. The diagnostic methods for small fiber neuropathy like Quantative Sensory Testing (QTS), Quantitative Sudomotor Axon Reflex Testing (QSART), Electromyography and Nerve-conduction Studies seem to have a lot of limitations and that is why skin biopsy is considered as the best diagnostic tool for SFN [20,21].
The procedure of punch skin biopsy is simple and easy to perform. It is commonly performed using a 3-mm punch under sterile technique. It can be taken from any part of the body, but the standard biopsy is performed $10 \mathrm{~cm}$ above the lateral malleolus to evaluate the loss of the most distal sensory endings typical of lengthdependent axonal neuropathy [22]. Another localization to perform biopsy is the upper thigh (20 cm distal to the iliac spine). All the guidelines how to perform skin biopsy were established in 2005 by European Federation of Neurological Societies [23]. The biopsy is performed under local anesthesia. The procedure is simple and easy to perform. It does not require a suture. The sample taken during the biopsy should be $2 \mathrm{~mm}$ thick to evaluate epidermis and surface of dermis. As indicated earlier, this procedure is relatively simple, however the disadvantages (i.e. invasiveness) of this technique also need to be mention. In addition to the unquestionable benefits of this procedure, not only performed in the diagnosis of SFN, complications are also possible. Infection is a not frequent, but a possible complication, especially in the risk group are diabetic patients, patients treated with immunosuppressants. What is obvious, this risk is increased by an inappropriate, nonsterile procedure execution. Both the benefits and adverse events of punch skin biopsy are presented in the Table $3[24,25]$.

The biopsy sample is later immunohistochemically stained with antibodies against protein gene product 9.5 (PGP 9.5). This protein belongs to the ubiquitin hydrolase family and is considered as a marker for peripheral nerve fibers and neuroendocrine cells. PGP 9.5 is used for over 30 years as a neuron-specific protein [26]. Most cutaneous fibers are unmyelinated, but in the dermis of a hairy skin there are 10\% smalldiameter myelinated fibers (A delta fibers). After the biopsy there is a fiber count performed. It includes counting of single axons that cross or originate at the epidermal-dermal junction. The result is expressed as the Epidermal Nerve Fiber Density (ENFD). According to European Federation of Neurological Societies reduced epidermal fiber density at the leg has a diagnostic value with $90 \%$ specificity and $82.6 \%$ sensitivity for small fiber neuropathy.

As it was mentioned above, not all patients suffering from fibromyalgia fulfill the criteria of small fiber neuropathy. Multiple studies confirm, that only about $40 \%-50 \%$ of patients 


\begin{tabular}{l} 
Table 3. Benefits and possible complications of the skin punch biopsy. \\
\begin{tabular}{|l|l|}
\hline Benefits / information & Possible complications \\
\hline Infections & Soreness or tenderness at the biopsy site \\
Precancerous tumors & Allergy to local anesthetic medications \\
Cancer growth & $-\quad$ itching \\
Benign tumors & $\begin{array}{l}\text { erythema } \\
\text { Eczema and Psoriasis }\end{array}$ \\
Small fiber neuropathy & Infection of the wound \\
& Scarring \\
\hline
\end{tabular} \\
\hline
\end{tabular}

with FM meets diagnostic criteria for small fiber neuropathy and has objective evidence of SFN, whereas 50\%-60\% does not [19,27]. It brings into question, how to extract the subgroup of FM patients with SFN. There was a study conducted at Massachusetts General Hospital from 2014 to 2015, with 39 FM patients, who underwent distal-leg skin biopsy and autonomic function testing to assess how many of them had SFN and to evaluate, whether there are some differences between these two fibromyalgia endophenotypes [27]. In the results obtained 14 (36\%) patients had test-confirmed SFN and 25 (64\%) did not. There was no difference in their pain severity, although the FM patients with SFN more often reported dysautonomia and paresthesias. The outcomes seem to be interesting, but we should remember, that the study was conducted on a small group of patients.

\section{Diagnosis of SFN-treatment implications}

The confirmation of SFN coexisting with FM influences treatment, although there are no strict recommendations. The approach to the treatment of SFN and FM should be multidirectional, aimed at elimination of pain, improvement of mood and improvement of physical activity.

As the first line treatment anticonvulsants, as gabapentin or pregabalin, are usually used, which act as inhibitors of $\alpha 2 \delta$ subunit-containing Voltage-Dependent Calcium Channels (VDCCs). There are reports of the effectiveness of newer antiepileptic drugs, like topiramate and zonisamide in the treatment of neuropathic pain $[28,29]$. Tricyclic antidepressants or serotoninnorepinephrine reuptake inhibitors have also been used. However, those medications combined with anti-epileptic drug - for example: gabapentin with an antidepressant, e.g. nortriptyline-may be more effective than monotherapy alone [30]. Although strong opioids are not recommended for SFN, nor for neuropathic pain not associated with neoplastic disease, weak opiates like tramadol are used in both these conditions [31]. In the treatment of neuropathic pain and FSN, it was shown, that in the case of a disruption of the sodium channels, the anti-arrhythmic drug, a sodium channel blocker - mexiletine, may have a positive effect [32]. Complementary methods of neuropathic pain treatment include a topical application of $5 \%$ lidocaine or $0.75 \%$ or $8 \%$ (patch) capsaicin and the use of acupuncture [33]. Data on other treatment methods, including over the counter supplements, are incomplete-however, it is worth noting, that the omega 3 fatty acids may be effective, especially in the treatment of patients with neuropathic pain with metabolic disorders, particularly in diabetes [34,35]. The relaxation, yoga or tai chi therapies may be used to supplement the treatment of SFN. Summarizing, the approach to therapy of both SFN and fibromyalgia should be holistic, with combination of drugs with different mechanism of action finding use.

\section{Conclusions}

The confirmation of SFN in fibromyalgia patients may lead to some implications with treatment, particularly in the therapy of neuropathic pain. So far fibromyalgia has been treated as vague disorder with unclear pathophysiology and it's diagnose has been based on subjective criteria. The robustness of studies of SFN in FM suggests that in some patients there is a fundamental component of peripheral tissue lesion. Additionally, small fiber neuropathy may be easily detected in FM patients what makes the diagnosis more objective. Furthermore, it arises to the conclusion that many of the symptoms seen in FM are likely to be immune mediated. Unfortunately, it can be applied only to selected patients and there is still more research needed to explain all the aspects of fibromyalgia's and SFN complexity. 


\section{References}

1. Hauser W, Ablin J, Fitzcharles MA et al. Fibromyalgia. Nat. Rev. Dis. Primers. 1, 15022 (2015).

2. Caro XJ, Winter EF. The role and importance of small fiber neuropathy in fibromyalgia pain. Curr. Pain. Headache. Rep. 19(12), 55 (2015).

3. Voortman M, Fritz D, Vogels OJM et al. Small fiber neuropathy: A disabling and underrecognized syndrome. Curr. Opin. Pulm. Med. 23(5), 447-457 (2017).

4. Levine TD. Small fiber neuropathy: Disease classification beyond pain and burning. J. Cent. Nerv. Syst. Dis. 10, 1179573518771703 (2018).

5. Sene D. Small fiber neuropathy: Diagnosis, causes, and treatment. Joint. Bone. Spine. (2017).

6. Hovaguimian A, Gibbons CH. Diagnosis and treatment of pain in small-fiber neuropathy. Curr. Pain. Headache. Rep. 15(3), 193-200 (2011).

7. Bakkers M, Faber CG, Hoeijmakers JG et al. Small fibers, large impact: Quality of life in small-fiber neuropathy. Muscle. Nerve. 49(3), 329-336 (2014).

8. Bacharach R, Lowden M, Ahmed A. Pyridoxine toxicity small fiber neuropathy with dysautonomia: A case report. J. Clin. Neuromuscul. Dis. 19(1), 43-46 (2017).

9. Gondim FAA, Barreira AA, Claudino R et al. Definition and diagnosis of small fiber neuropathy: consensus from the Peripheral Neuropathy Scientific Department of the Brazilian Academy of Neurology. Arq. Neuropsiquiatr. 76(3), 200-208 (2018).

10. Gibbons C, Freeman R. The evaluation of small fiber function-autonomic and quantitative sensory testing. Neurol. Clin. 22(3), 683-702 (2004).

11. Lauria G, Lombardi R. Small fiber neuropathy: is skin biopsy the holy grail? Curr. Diab. Rep. 12(4), 384-392 (2012).

12. Hauser W, Jung E, Erbsloh-Molle B et al. Validation of the fibromyalgia survey questionnaire within a crosssectional survey. PloS. One. 7(5), e37504 (2012).

13. Jones GT, Atzeni F, Beasley M et al. The prevalence of fibromyalgia in the general population: A comparison of the American College of Rheumatology 1990, 2010, and modified 2010 classification criteria. Arthritis. Rheumatol. 67(2), 568-575 (2015).

14. Oaklander AL, Klein MM. Evidence of small-fiber polyneuropathy in unexplained, juvenile-onset, widespread pain syndromes. Pediatrics. 131(4), e10911100 (2013).

15. Oaklander AL, Herzog ZD, Downs HM et al. Objective evidence that small-fiber polyneuropathy underlies some illnesses currently labeled as fibromyalgia. Pain. 154(11), 2310-2316 (2013).

16. Caro XJ, Winter EF. Evidence of abnormal epidermal nerve fiber density in fibromyalgia: clinical and immunologic implications. Arthritis. Rheumatol. 66(7), 1945-1954 (2014).

17. Giannoccaro MP, Donadio V, Incensi A et al. Small nerve fiber involvement in patients referred for fibromyalgia. Muscle. Nerve. 49(5), 757-759 (2014).
18. Kosmidis ML, Koutsogeorgopoulou L, Alexopoulos $\mathrm{H}$ et al. Reduction of intraepidermal nerve fiber density (IENFD) in the skin biopsies of patients with fibromyalgia: A controlled study. J. Neurol. Sci. 347(12), 143-147 (2014)

19. Levine TD, Saperstein DS. Routine use of punch biopsy to diagnose small fiber neuropathy in fibromyalgia patients. Clin. Rheumatol. 34(3), 413-417 (2015).

20. England JD, Gronseth GS, Franklin G et al. Evaluation of distal symmetric polyneuropathy: the role of autonomic testing, nerve biopsy, and skin biopsy (an evidence-based review). Muscle. Nerve. 39(1), 106-115 (2009).

21. Saperstein DS, Levine TD, Levine M. Usefulness of skin biopsies in the evaluation and management of patients with suspected small fiber neuropathy. Int. J. Neurosici. 123(1), 38-41 (2013).

22. Hays AP. Utility of skin biopsy to evaluate peripheral neuropathy. Curr. Neurol. Neurosci. Rep. 10(2), 101107 (2010).

23. Lauria G, Hsieh ST, Johansson O et al. European federation of neurological societies/peripheral nerve society guideline on the use of skin biopsy in the diagnosis of small fiber neuropathy. Report of a joint task force of the European Federation of Neurological Societies and the Peripheral Nerve Society. Eur. J. Neurol. 17(7), 903-912, e944-909 (2010).

24. Nischal U, Nischal Kc, Khopkar U. Techniques of skin biopsy and practical considerations. J. Cutan. Aesthet. Surg. 1(2), 107-111 (2008).

25. Abhishek K, Khunger N. Complications of skin biopsy. J. Cutan. Aesthet. Surg. 8(4), 239-241 (2015).

26. Lundberg LM, Alm P, Wharton J. Protein gene product 9.5 (PGP 9.5). A new neuronal marker visualizing the whole uterine innervation and pregnancy-induced and developmental changes in the guinea pig. Histochemistry. 90(1), 9-17 (1988).

27. Lodahl M, Treister R, Oaklander AL. Specific symptoms may discriminate between fibromyalgia patients with vs without objective test evidence of small-fiber polyneuropathy. Pain. Rep. 3(1), e633 (2018).

28. Tavee JO. Office approach to small fiber neuropathy. Cleve. Clin. J. Med. 85(10), 801-812 (2018).

29. Hasegawa H. Utilization of zonisamide inpatients with chronic pain orepilepsy refractory to othertreatments: a retrospective,open label, uncontrolled studyin a VA hospital. Curr. Med. Res. Opin. 20(5), 577-580 (2004).

30. Gilron I, Bailey JM, Tu D. Nortriptyline and gabapentin, alone and in combination for neuropathic pain: A double-blind, randomised controlled crossover trial. Lancet. 374(9697), 1252-1261 (2009).

31. Ho TW, Backonja M, Ma J et al. Efficient assessment of neuropathic pain drugs in patients with small fiber sensory neuropathies. Pain. 141(1-2), 19-24 (2009).

32. Challapalli V, Tremont-Lukats IW, McNicol ED et al. Systemic administration of local anesthetic agents to relieve neuropathic pain. Cochrane Database of Systematic Reviews 4, CD003345 (2005).

33. Attal N, Cruccu G, Baron R et al. EFNS guidelines on the pharmacological treatment of neuropathic pain: 2010 revision. Eur. J. Neurol. 17(9), 1113-e1188 (2010). 
34. Lewis EJ, Perkins BA, Lovblom LE et al. Effect of omega-3 supplementation on neuropathy in type 1 diabetes: a 12-month pilot trial. Neurol. 88(24), 2294 2301 (2017).
35. Ko GD, Nowacki NB, Arseneau L et al. Omega-3 fatty acids for neuropathic pain: case series. Clin. J. Pain. 26(2), 168-172 (2010) 